\title{
Validação de cartilha educativa para a promoção de conforto de familiares da pessoa no intraoperatório
}

\author{
Educational booklet validation for the promotion of comfort to relatives of people in intraoperative \\ period
}

Validación de um folleto educativo para promover la comodidad de los familiares de la persona durante el período intraoperatorio

Maryana Carneiro de Queiroz Ferreira

ORCID: https://orcid.org/0000-0002-5711-4485

Universidade Estadual de Feira de Santana, Brasil E-mail: maryanacarneiro00@gmail.com

Marluce Alves Nunes Oliveira

ORCID: https://orcid.org/0000-0002-9109-0106

Universidade Estadual de Feira de Santana, Brasil E-mail: milicialves@yahoo.com.br

Kátia Santana Freitas

ORCID: https://orcid.org/0000-0002-0491-6759

Universidade Estadual de Feira de Santana, Brasil E-mail: freitaskatia@yahoo.com.br

Elaine Guedes Fontoura

ORCID: https://orcid.org/0000-0001-7423-0464 Universidade Estadual de Feira de Santana, Brasil E-mail: egfontoura@uefs.br

Joselice Almeida Gois

ORCID: https://orcid.org/0000-0001-8870-3509

Universidade Estadual de Feira de Santana, Brasil E-mail: joselice.gois@ hotmail.com

Pollyana Pereira Portela

ORCID: https://orcid.org/0000-0002-6840-4533

Universidade Estadual de Feira de Santana, Brasil E-mail: pollyana.pportela@gmail.com

Tayara de Oliveira Vitoria

ORCID: https://orcid.org/0000-0002-0282-0163

Universidade Estadual de Feira de Santana, Brasil

E-mail: tayaravitoria@gmail.com

Lorraine Alves Souza Santos

ORCID: https://orcid.org/0000-0001-5488-8533

Universidade Estadual de Feira de Santana, Brasil

E-mail: lorrainealves1992@gmail.com

Ayla Melo Cerqueira

ORCID: https://orcid.org/0000-0001-7432-484X

Universidade Estadual de Feira de Santana, Brasil

E-mail: aylacerqueira12@gmail.com

Íris Cristy da Silva e Silva

ORCID: https://orcid.org/0000-0002-5453-8020

Universidade Estadual de Feira de Santana, Brasil

E-mail: iriscristy22@gmail.com

\begin{abstract}
Resumo
Objetivo: Validar a cartilha educativa para a promoção de conforto dos familiares da pessoa no período intraoperatório. Metodologia: Trata-se de um estudo metodológico, com abordagem quantitativa e qualitativa, realizado em três etapas: Diagnóstico situacional e levantamento bibliográfico; Construção da cartilha educativa e Validação da cartilha educativa com nove juízes (experts). A avaliação ocorreu em duas etapas. Para análise dos dados quantitativos utilizou-se a taxa de concordância proposta por Domingues e colaboradores, e para os dados qualitativos utilizou-se a análise de conteúdo proposta por Bardin. Resultados: No primeiro momento 84,6\% dos tópicos abordados obtiveram taxa de concordância insatisfatória, abaixo de $80 \%$, na segunda rodada de avaliação, após alterações na cartilha, apenas um item não obteve taxa de concordância de $100 \%$. Na análise qualitativa, a cartilha foi considerada ferramenta importante para a promoção de conforto e orientações necessárias para o processo
\end{abstract}


de acompanhamento, e apontado possibilidades e limites para sua implementação. Conclusão: A cartilha foi considerada válida para a promoção de conforto dos familiares da pessoa no período intraoperatório.

Palavras-chave: Estudo de validação; Tecnologia educacional; Cuidados de enfermagem; Família; Período intraoperatório.

\begin{abstract}
Goals: Validate the educational booklet for the comfort promotion of the relatives of people in intraoperative period. Methodology: It's a methodological study based on the "Recommendations for the Conducting and Reporting of Delphi Studies", made from October of 2019 to August of 2020, in a big public hospital in Bahia, Brazil. The study was developed in three stages: Situational diagnostic and bibliographic survey, Educational booklet development and Educational booklet validation by the experts. The evaluation happened in two moments: For the quantitative data analysis was used the concordance rate proposed by Domingues and collaborators, and for the qualitative data was used the analysis proposed by Bardin. Results: At the first moment 84,6\% of the approached topics had an unsatisfactory concordance rate, under $80 \%$. At the second round,after changes in the booklet, only one item didn't have concordance rate of $100 \%$. On the qualitative analysis, the booklet was considered a important tool for the comfort promotion and necessary guidance for the monitoring process, and pointing possibilities and limits for it implementation. Conclusion: The booklet was considered valid when it comes to comfort promotion to the relatives of people in intraoperative period.
\end{abstract}

Keywords: Validation study; Educational technology; Nursing care; Family; Intraoperative period.

\title{
Resumen
}

Objetivo: Validar el folleto educativo para promover la comodidad de los familiares de la persona durante el periodo intraoperatorio. Metodología: Se trata de un estudio metodológico, con enfoque cuantitativo y cualitativo, realizado entres etapas: Diagnóstico situacional y levantamiento bibliográfico; Construcción del cuadernillo educativo y Validación del cuadernillo educativo con nueve jueces (expertos). La evaluación se realizó en dos etapas. Para el análisis de los datos cuantitativos se utilizó la tasa de acuerdo propuesta por Domingues y colaboradores y para los datos cualitativos se utilizó el análisis de contenido propuesto por Bardin. Resultados: En el primer momento, 84.6\% de los temas tratados tuvieron una tasa de acuerdo insatisfactoria, inferior al 80\%, en la segunda ronda de evaluación, después de cambios en el cuadernillo, solo un ítem no obtuvo una tasa de acuerdo del 100\%. En el análisis cualitativo, el cuadernillo fue considerado una herramienta importante para promover la comodidad y la orientación necesaria para el proceso de seguimiento, y señaló posibilidades y límites para su implementación. Conclusión: el folleto fue considerado válido para promover la comodidad de los familiares de la persona durante el period intraoperatorio.

Palabras clave: Estudio de validación; Tecnología educacional; Atención de enfermería; Familia; Periodo intraoperatorio.

\section{Introdução}

O conforto é considerado uma necessidade humana básica, proposta essencial e prioritária, com um objetivo terapêutico para atenção de cada pessoa, a fim de promover bem-estar (Cardoso et al., 2019). Nessa perspectiva, no ambiente cirúrgico, o conforto precisa ser implementado para além da pessoa que está passando por processo diagnostico-terapêutico, e abordar aspectos relevantes para a família (Zhao et al., 2016), que vivencia os sentimentos de ansiedade, expectativas e medo junto com a pessoa que se encontra no período intraoperatório.

O período intraoperatório, inicia com a transferência da pessoa para a sala de operação e termina com a conclusão do procedimento cirúrgico e transferência da pessoa para a sala de recuperação pós-anestésica (Escobar et al., 2018). Importante ressaltar que, a pessoa adoecida pode ser encaminhada de uma unidade de internação ou de unidade de emergência.

Logo, a inclusão de familiares para a promoção de conforto durante o período intraoperatório pode contribuir para que se sintam seguros e confiantes em relação a cirurgia e os cuidados prestados, tanto durante a cirurgia, quanto na recuperação cirúrgica (Hamester et al., 2016). Família é um grupo de indivíduos ligados por laços de sangue e laços sociais, que se relacionam cotidianamente, produzindo significados e práticas associadas a saúde, doença e cuidado, além de contemplar as diversidades de relações na sociedade (Queiroz et al., 2015).

Diante disso, o conforto para os familiares da pessoa em processo cirúrgico se inicia na consulta pré-operatória, onde os profissionais podem fornecer informações do procedimento a ser realizado e ofertar cuidados, permitindo a promoção de conforto e melhor recuperação da pessoa que vai passar pela cirurgia (Hamester et al., 2016). Além do mais, no período 
intraoperatório podem garantir conforto por meios de produções educativas: folder, álbum seriado, cartilhas, dentre outros. A disponibilização de folhetos e cartilhas é possível promover informações e orientações escritas a familiares no contexto hospitalar, que permite adesão as orientações, além de ser valido para minimizar os desconfortos vivenciados pelos familiares, elevando o bem-estar físico, psicológico, espiritual, relacionamento social e enfrentamento das experiências que podem ser vividas (Muniz et al., 2019).

A cartilha educativa para os familiares de pessoas no período intraoperatório, possibilita ao profissional ou estudante da área de saúde a oportunidade de oferecer informações e orientações pertinentes a unidade do centro cirúrgico, procedimento que estar sendo realizado, situação do seu ente, enquanto aguardam a cirurgia, assim realiza-se a promoção de conforto. E para garantir a eficácia esperada desses materiais educativos, é preciso que os mesmos sejam validados por juízes (experts) como tecnologias eficazes para o cuidado em saúde, de forma que o cuidado seja promovido com qualidade e eficiência (Bezerra et al., 2018).

Em relação a importância da cartilha educativa na promoção de conforto de familiares que aguardam seu ente no intraoperatório nos levou a seguinte inquietação: Como validar a cartilha educativa para promoção de conforto aos familiares da pessoa no período intraoperatório?

Consideramos este estudo relevante, vez que são escassas produções de construção e validação de materiais educativos que visem a promoção de conforto dos familiares da pessoa no período intraoperatório. Outra possibilidade está relacionada a reflexão de estudantes e profissionais da área da saúde para a promoção de conforto aos familiares de pessoas hospitalizadas, visto que o cuidado precisa ser integral e para além da pessoa adoecida.

O objetivo deste estudo é validar a cartilha educativa para a promoção de conforto dos familiares da pessoa no intraoperatório.

\section{Metodologia}

Trata-se de um estudo metodológico, baseado na Recommendation for the Conducting and Reporting of Delphi Studies (CREDES) (Jünger et al., 2017), realizado no período de outubro de 2019 a agosto de 2020, em hospital público de grande porte no interior da Bahia, Brasil, que possui unidade do Centro Cirúrgico (CC) para cirurgias eletivas e emergenciais. A pesquisa foi realizada utilizando a Técnica Delphi para validação da cartilha educativa, considerada um método acessível, por possibilitar uma avaliação de um instrumento por especialistas com experiência clínica e/ou acadêmica, realizada através de várias rodadas a fim de atingir consenso e qualidade do instrumento avaliado (Jünger et al., 2017).

A pesquisa foi desenvolvida em três etapas: 1. Diagnóstico situacional e levantamento bibliográfico; 2. Construção da cartilha educativa; 3. Validação da cartilha educativa com juízes (experts).

A primeira etapa consistiu no diagnóstico situacional realizado por meio de diálogo informal com 08 familiares que se encontravam na sala de espera do CC de um hospital público do interior da Bahia, Brasil em 2019, com o objetivo de obter as percepções de conforto e desconfortos enfrentados no processo de acompanhamento de seu familiar, o diálogo ocorreu de forma livre, espontânea e interativa entre os familiares, onde os mesmos foram relatando suas experiências e o que gostariam de conhecer em relação ao $\mathrm{CC}$ e o procedimento cirúrgico, a fim de que permanecessem de forma mais confortável.

Posteriormente, guiada pela demanda dos familiares das pessoas no intraoperatório, procedeu-se um levantamento bibliográfico de estudos completos dos últimos cinco anos, em português, inglês e espanhol, sobre a promoção de conforto dos familiares da pessoa no período intraoperatório, cuidado aos familiares que aguardam a espera da cirurgia, processo de acompanhamento de pessoas hospitalizadas, objetivando construir a cartilha baseada em evidências científicas.

A segunda etapa, construção da cartilha educativa, consistiu na junção das partes textuais, em linguagem simples e de fácil entendimento, e as ilustrações, que foram retiradas do Google imagens e devidamente referenciadas, tendo como critério 
de seleção que as imagens fossem desenhos que se aproximassem da realidade encontrada pelos familiares no CC. Para a construção, tomamos como base as vivências de familiares da pessoa no intraoperatório e a revisão bibliográfica, visando promover conforto e conhecimento aos familiares. A cartilha educativa foi construída através do Publisher do Micrsoft office 2016, e intitulada "Promoção de conforto aos familiares de pessoas no intraoperatório".

$\mathrm{Na}$ terceira etapa, contou com a avaliação de nove juízes (experts), sendo enfermeiros com especialização e experiência em CC. O processo de validação ocorreu por meio de um instrumento com questões abertas e fechadas autoaplicáveis, realizada em dois momentos para permitir a eficácia do material educativo, sendo verificado se os quesitos abordados e a aparência estavam claros e pertinentes aos familiares da pessoa no período intraoperatório.

Os juízes para a avaliação, foram pesquisados por meio do currículo lattes, com os descritores "Enfermeiros de centro cirúrgico", "especialização em centro cirúrgico", "especialista em centro cirúrgico" e "Enfermeiro do bloco cirúrgico", do Brasil. Selecionados dois filtros, o que refere à especialização acadêmica, e o relacionado a área de atuação, aplicado o item que diz respeito a Enfermagem.

Foram encontrados 7521 currículos, desses, após leitura e análise, foram selecionados 54 juízes, que se enquadravam nos critérios de inclusão: Ser enfermeiro com especialização em CC, ter prática em CC (de preferência atual), ter participação em cursos e/ou eventos relacionados ao $\mathrm{CC}$, ter pesquisas publicadas com temáticas relacionadas ao CC e ter currículo lattes atualizado com intuito de possibilitar avaliação precisa da cartilha educativa, e como critérios de exclusão: Não possuir pósgraduação em CC e o currículo lattes estar atualizado a mais de um ano. Desses 54 juízes pré selecionados, 11 aceitaram o convite, em seguida foi enviado a cartilha educativa com o instrumento de validação. Participaram da avaliação da cartilha 09 juízes.

O contato com os especialistas ocorreu pelo endereço eletrônico disponibilizado no currículo lattes, sendo enviada a carta convite explicando o objetivo da pesquisa e a importância de sua avaliação, em anexo o Termo de Consentimento Livre e Esclarecido (TCLE), e solicitado que na aceitação de participação como especialista assinassem e enviassem por e-mail.

A avaliação dos especialistas ocorreu em duas etapas. Na primeira utilizou-se um instrumento com questões fechadas, construído pelo Microsoft Word. Foi utilizado a escala tipo Likert, sendo 0 para discordo, 1 para concordo parcialmente e 2 para concordo totalmente (Leite et al., 2018), considerando os critérios de clareza e pertinência do conteúdo elaborado. A avaliação ocorreu considerando itens gerais e específicos da cartilha.

Adicionalmente, foram realizadas perguntas abertas pela pesquisadora que levaram a análise qualitativa da validação, sendo elas: 1. Em sua opinião a cartilha educativa elaborada pode promover conforto aos familiares da pessoa no intraoperatório? 2. Fale-me das possibilidades e limites para a cartilha educativa promover conforto aos familiares de pessoas no intraoperatório. 3. Comente sobre o que deve ser acrescentado/retirado na cartilha educativa para ser compreendido pelos familiares de pessoas no intraoperatório. Dos 09 juízes que participaram da avaliação da cartilha, apenas 07 responderam as questões subjetivas.

Após essa análise dos juízes, a cartilha foi reavaliada e as sugestões para as modificações necessárias ao seu aperfeiçoamento foram realizadas, para novamente ser avaliada pelos especialistas.

A segunda versão da cartilha educativa foi enviada para os juízes para que fizessem nova avaliação, e verificar se após as alterações a cartilha encontrava-se clara e pertinente ao público alvo, sendo que nessa etapa foi devolvido para os especialistas apenas o instrumento com questões fechadas. Dos 09 juízes que avaliaram a cartilha na primeira etapa, apenas 07 enviaram as respostas da segunda rodada para validação. Entretanto, consideramos suficiente para obtenção de índices para a validação da cartilha.

Para análise dos dados quantitativos, referente a resposta ao instrumento de avaliação, utilizou-se a taxa de concordância, que visa verificar a porcentagem de juízes que estão em concordância dos instrumentos analisados e nos 
aspectos abordados nos mesmos. Para obter a taxa de concordância dos itens foi dividido o número de juízes que responderam com concordo totalmente sobre número total de juízes (Ribeiro et al., 2017). Essa taxa, foi interpretada considerando um resultado maior ou igual a $80 \%$, indicando que os itens estavam apropriados e, portanto, válidos (Domingues et al., 2016). Os itens que tiveram uma taxa de concordância menor que $80 \%$ foram alterados ou excluídos a fim de possuir uma concordância maior.

Para análise dos dados qualitativos, referente as respostas do juízes na primeira etapa de avaliação do material educativo, utilizou a técnica de Análise de Conteúdo, proposta por Bardin (2016), trata-se de uma análise sistematizada, organizados através de três etapas, a pré-análise, que visa a organização, nela foi realizada leitura flutuante para permitir uma exploração do material, escolha do material que foi analisado, organizado e sistematizado em indicadores e reunindo recortes dos texto o que contribuiu para a categorização, a segunda fase é a exploração do material, onde foram realizadas operações de codificação, decomposição ou enumeração em função de regras previamente formuladas, e por último foi realizado o tratamento dos resultados obtidos e interpretação, sendo feitas inferências e interpretações baseadas no objetivo propostos.

Este estudo foi aprovado pelo Comitê de Ética e Pesquisa da Universidade Estadual de Feira de Santana, sob parecer n 2.277.332, atendendo os direitos humanos contidos na Resolução 466/12 do Conselho Nacional de Saúde (Brasil, 2012). Os participantes (juízes) assinaram o Termo de Consentimento Livre e Esclarecido (TCLE), mediante orientações dos objetivos e finalidades da pesquisa. Para garantir o anonimato dos juízes e protegê-los quanto à reparação posterior, foram identificados com nomes de pedras preciosas: Cristal, Esmeralda, Pérola, Ágata, Rubi, Blenda, Jade, Safira e Olivin.

\section{Resultados}

Os resultados são apresentados em dois momentos. O primeiro a análise quantitativa, pela taxa de concordância (Domingues et al., 2016), e o segundo, a análise qualitativa pela análise de conteúdo (Bardin, 2016).

Após a análise das entrevistas foi estruturado os resultados em duas etapas, a primeira com a validação da cartilha educativa e a segunda através de categorias empíricas encontradas: Cartilha educativa como forma de promover conforto no período intraoperatório; Possibilidades e limites da cartilha educativa promover conforto.

A validação da cartilha contou com a participação de nove juízes (experts), enfermeiros que atuam em CC, no Brasil, dentre eles, oito do sexo feminino e um do sexo masculino. Quanto a formação, todos possuem especialização em enfermagem em CC, sete juízes possuem mestrado, apenas quatro com teses com objeto em CC, e três possuem doutorado, apenas dois com objetos de CC. Quanto ao tempo de atuação profissional em CC, variou de 2 anos até 20 anos. Todos possuem pesquisas e participaram em cursos e/ou eventos relacionados ao CC.

\subsection{Validação da cartilha educativa}

A avaliação da cartilha educativa ocorreu em duas etapas, primeiro foram avaliados os itens gerais, principalmente no que se refere à estrutura e apresentação da cartilha, e posteriormente os itens individuais e específicos de cada tópico abordado.

Na primeira avaliação pelos juízes (Tabela 1), no que concerne avaliação dos itens gerais da cartilha, cinco quesitos (62,5\%) obtiveram valor menor que o ponto de corte estabelecido (80\%), sendo eles, título (77,78\%), layout (66,67\%), itens abordados na cartilha $(55,56 \%)$, linguagem abordada $(66,67 \%)$ e se a cartilha continha informações importantes sobre o conforto aos familiares que aguardam as pessoas no intraoperatório $(77,78 \%)$. 
Tabela 1. Primeira análise dos itens da cartilha educativa pelos juízes.

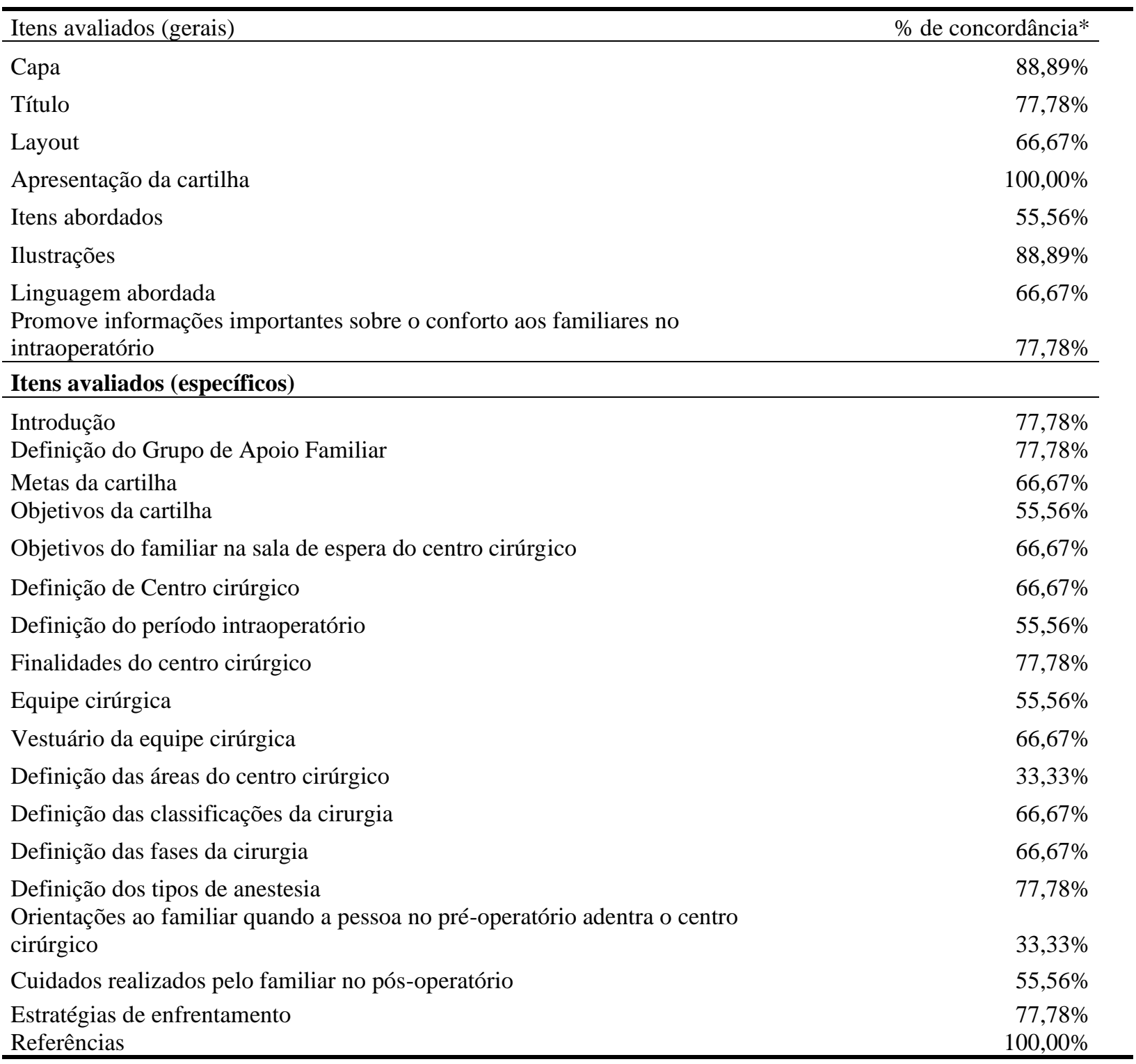

*\% de concordância: Taxa de concordância. Fonte: Elaboração própria

Nessa perspectiva, nos itens que tiveram uma taxa de concordância menor que $80 \%$, foram realizadas alterações, conforme as sugestões dos juízes, a fim de que possibilitasse melhor apresentação e compreensão pelos familiares da pessoa no intraoperatório.

As principais alterações nos itens gerais avaliados encontram-se na linguagem abordada que, para os juízes, estava pouco direcionada ao público-alvo e dessa forma, afetando os quesitos gerais que obteve baixa taxa de concordância. Ademais, também foi sugerido que a cartilha abordasse itens referentes ao período perioperatório, com o objetivo de promover conforto para mais pessoas. Esses comentários feitos pelos juízes justificam também o quesito itens abordados na cartilha, que obteve uma taxa de concordância menor na avaliação geral (55,56\%), pois segundo os juízes estavam direcionados a equipe de saúde, como também sugeriram acrescentar alguns quesitos, como a abordagem da cirurgia segura.

Na linguagem abordada, além das sugestões mencionadas acima, também foi sugerido a substituição de palavras técnicas, visando uma linguagem mais clara aos familiares. No layout, foi sugerido a retirada de corações no fundo das páginas, visto que afeta a concentração do leitor, e o melhor uso das cores e espaçamentos utilizados. 
Quanto ao título (77,78\%), ele alterado de "Promoção de conforto aos familiares de pessoas no intraoperatório" para "Promoção de conforto aos familiares de pessoas em processo cirúrgico", por considerar melhor para a compreensão dos familiares, mediante sugestão de juízes.

A apresentação da cartilha obteve uma taxa de concordância de 100\%, demonstrando que estava pertinente para os familiares da pessoa no intraoperatório. Além disso, a capa e as ilustrações apresentadas (88,89\%), estavam adequadas para os familiares, e capazes de possibilitar uma aproximação com o momento vivido.

No que se refere à avaliação específica, os tópicos Definição das áreas do centro cirúrgico e Orientações ao familiar quando a pessoa no pré-operatório adentra o centro cirúrgico, foram os itens que possuíram menor taxa de concordância $(33,33 \%)$.

No que concerne às áreas de $\mathrm{CC}$, dois juízes mencionaram que a informação era desnecessária ao familiar. Deles, um juiz marcou 0 na escala e o outro 1, realizando apenas uma sugestão de retirada. Além do mais, foi sugerido realizar a troca das nomenclaturas das divisões do CC, a fim de permitir que a cartilha educativa apresentasse referência atual e correção de ortografia.

Referente às orientações prestadas ao familiar quando a pessoa no pré-operatório adentra no $\mathrm{CC}$, foi mencionado por um juiz, que a linguagem abordada estava técnica e pouco direcionada ao público-alvo, além de conter informações sobre instrumentos hospitalares, que as pessoas podem estar em uso e que para os familiares são desconhecidos, como tubos, sondas e drenos. Também foi sugerido acrescentar na retirada de acessórios, o piercing, devido ao risco que pode causar no paciente, além de não entrar no CC usando maquiagem e informar se o paciente possui alergia.

Os objetivos da cartilha tiveram taxa de concordância baixa (55,56\%), que conforme os juízes, não se apresentavam de forma clara, sendo confundido se os objetivos eram da cartilha ou do grupo de apoio familiar. Ademais, foi descrito novamente a relevância da promoção de conforto aos familiares no período perioperatório ao invés do intraoperatório, sendo as mesmas sugestões aplicadas as metas que foram abordadas $(66,67 \%)$.

$\mathrm{Na}$ definição do período intraoperatório (55,56\%), um juiz discordou da informação presente, enquanto outros fizeram correções de erros ortográficos e sugeriram o acréscimo de explicação da Sala de Recuperação Pós-Anestésica (SRPA), pois para os familiares é um ambiente desconhecido.

Em relação a equipe cirúrgica $(55,56 \%)$, foi sugerido e orientado quanto a troca do termo por equipe do centro cirúrgico, pois, conforme o juiz, equipe cirúrgica se refere apenas aos profissionais que fazem parte do procedimento cirúrgico. Foi sugerido incluir gêneros nos nomes dos profissionais e retirar "e demais profissionais", visto que fica subtendido para o público leigo. Ainda relacionado à equipe cirúrgica, no que diz respeito ao vestuário (66,67\%), foi sugerido incluir imagens referente aos Equipamentos de Proteção Individual (EPIs), acrescentado e explicado sobre roupa privativa.

Nos cuidados realizados pelo familiar no pós-operatório, $(55,56 \%)$ consideram que as orientações sejam passadas de forma mais clara e objetiva, além de abordar o cuidado no pós-operatório hospitalar, visando incluir mais familiares e substituir palavras técnicas por habituais para facilitar a compreensão.

No item de definição do CC $(66,67 \%)$, os três juízes que concordaram parcialmente com essa informação, fizeram a mesma sugestão, retirada do parágrafo que diz que o CC é um ambiente causador de desconfortos e sentimentos desagradáveis, portanto, foi retirada essa informação. Quanto aos tipos de anestesia (77,78\%), foi sugerido acrescentar a sedação e adicionar informação no início da cartilha.

Finalidades do CC (77,76\%) e classificações da cirurgia (66,67\%), mesmo não possuindo taxa de concordância baixa, comparado a outros itens avaliados, foram retiradas da cartilha educativa por considerar que, após a sugestão dos juízes, essas informações eram desnecessárias aos familiares.

Os objetivos do familiar na sala de espera do CC $(66,67 \%)$ e definições das fases da cirurgia $(66,67 \%)$, embora 
tenham tido taxa de concordância menor que o ponto de corte, os ajustes realizados se basearam na correção de erros de ortografia e pontuação.

A introdução $(77,78 \%)$, definição do grupo de apoio familiar $(77,78)$ e referências $(100 \%)$, apresentaram sugestões mínimas, que não altera o conteúdo.

Após alterações da cartilha, segundo as sugestões dos juízes, foi enviado novamente para eles a fim de que reavaliassem os quesitos abordados. Na segunda etapa de avaliação da cartilha, apenas um item não obteve taxa de concordância de $100 \%$, sendo ele, vestuário da equipe do CC (85,71\%), que para o juiz, faz-se necessário explicar melhor a roupa privativa, pois mesmo com a imagem ilustrativa da mesma, os familiares podem permanecer com dúvidas.

Além disto, todos os demais itens avaliados tiveram taxa de concordância de $100 \%$, demonstrando que a cartilha educativa após modificações sugeridas pelos juízes se apresenta válida, além disso clara, objetiva e direcionada ao público alvo, sendo capaz de atingir os objetivos propostos.

\subsection{Cartilha educativa como forma de promover conforto no período intraoperatório}

Nesta categoria os juízes consideraram a cartilha como uma ferramenta importante para a promoção de conforto aos familiares da pessoa no período intraoperatório por conter informações relevantes.

Sim. A cartilha é um importante instrumento para oferecer conforto aos familiares. Linguagem clara e layout atrativo. (Jade)

Considero a cartilha apresentada uma ferramenta válida. No momento que estamos aguardando um parente ou amigo submetido a procedimento cirúrgico. [...] A cartilha pode ser uma ferramenta a auxiliar nesse processo. (Esmeralda)

Sim. Só o fato de se apresentar o passo a passo que o paciente vai percorrer já traz para o familiar um certo conforto. (Cristal)

Para Jade e Esmeralda, a cartilha é um importante instrumento para a promoção de conforto dos familiares da pessoa no período intraoperatório. Esmeralda ainda acrescenta que a cartilha educativa pode auxiliar no processo de espera da cirurgia do seu ente. Cristal considera como importante, pois apresenta as fases que o paciente vai percorrer, mantendo o familiar informado.

As informações presentes na cartilha educativa são essenciais para os familiares que aguardam a realização da cirurgia do seu ente na sala de espera, sendo considerada como forma de promover bem-estar e satisfazer as necessidades básicas dos familiares e dessa forma promover conforto, como relata Pérola e Rubi.

Sim, em minha opinião, esta cartilha pode levar aos familiares, informações necessárias para manter-se em seu estado de equilíbrio até que seu familiar receba alta do centro cirúrgico... (Pérola)

A cartilha está bem elaborada, apresentando informações pertinentes e importantes para promoção do conforto aos familiares de pessoas no intraoperatório. Considero muito válida... (Rubi)

Além disso, atrelado a disponibilização da cartilha educativa, o profissional também precisa estar presente, para auxiliar na promoção de conforto dos familiares, relata Pérola. 
O conforto neste caso, será mais enfatizado pelo profissional, que mesmo diante da rotina exaustiva e excessiva de um centro cirúrgico, deverá encontrar um tempo para confortar os familiares de forma que os mesmos se sintam seguros apesar de não estarem presentes no momento do ato anestésico-cirúrgico. (Pérola)

Para Pérola em sua fala, a cartilha é um instrumento importante, mas para o conforto ser promovido de forma eficaz, o profissional de saúde precisa estar presente para auxiliar nesse processo.

\subsection{Possibilidades e limites da cartilha educativa promover conforto}

Conforme relatado pelos juízes a cartilha educativa possui possibilidades de ser disponibilizada para os familiares da pessoa no intraoperatório, como uma forma de fornecer informações e serem aceitas, além de ser importante para promover conforto, distração e auxiliar em momentos de ansiedade que podem emergir nesse período.

Acredito que esta cartilha, tem grandes possiblidades de levar aos familiares e pacientes informações relevantes ao período intraoperatório. Algumas mudanças devem ser realizadas, a fim de contribuir para um melhor entendimento... (Pérola)

Possibilidades: aceitar ou não os passos da cartilha pelos familiares, porém terão que aceitar as regras institucionais. (Cristal)

Possibilidades: distração e conforto para os familiares nesse momento de ansiedade. (Jade).

Possibilidade auxiliar a família com ansiedade durante o período de espera. (Blenda)

Para Pérola, as possibilidades da cartilha educativa, é levar às famílias informações pertinentes ao momento vivenciando, sendo necessário revisões do material para torná-lo mais adequado. Cristal se refere a possibilidade de aceitação ou não das informações contidas na cartilha e para Jade e Blenda, a cartilha educativa é importante para minimizar a ansiedade vivenciada.

A cartilha educativa possui limites para sua implementação, sendo o grau de escolaridade um dos principais a ser enfrentados, visto que nem todas as pessoas são alfabetizadas ou compreendem a linguagem que foi abordada.

Limites: grau de escolaridade do acompanhante (Cristal)

Limites: particulares de cada familiar, como o baixo nível de escolaridade (analfabetismo), ou mesmo o não interesse pela leitura. (Jade)

Infelizmente a cartilha não alcançará todos os públicos por conta dos termos técnicos, mas será muito bem aproveitada por que tem um pouco de conhecimento, visto que falar de centro cirúrgico e não usar termos técnicos é quase que impossível. (Ágata)

Cristal, Jade e Ágata, consideram o grau de escolaridade como um limite, visto que, pode se encontrar pessoas com baixo nível de escolaridade ou analfabetos, que não compreenderam a linguagem abordada, além de pessoas que não se interessem pela leitura.

Como limites ainda, são os períodos do processo cirúrgico, onde foi relatado que os familiares durante o perioperatório precisam de conforto, inclusive no pós-operatório, visto que, algumas pessoas podem ir para a UTI, o que 
ocasiona mais desconforto nos familiares.

[...] Como limitação para promoção do conforto dos familiares, destaco a questão dos períodos. Penso que a cartilha possa contribuir não apenas para o conforto dos familiares no intraoperatório (Rubi)

Um dos limites seria para o caso de pacientes com provável ida para UTI após o procedimento. Não constam informações para essa fase... (Esmeralda)

Rubi ressalta que a promoção de conforto deve ser realizada em todo o período perioperatório, pois em todo esse período são vividos sentimentos desagradáveis. Esmeralda relata a importância de abordar informações sobre a possível ida a UTI.

\section{Discussão}

A construção e validação de cartilha educativa é considerada importante para promover informações e conforto para familiares que aguardam seus entes no tratamento realizado em instituição de saúde. $\mathrm{O}$ uso de tecnologias educacionais impressa, como é o caso da cartilha educativa, é considerada uma ferramenta viável para educação em saúde, pois permite a oferta de informações pertinentes para a população, capaz de sensibilizá-las (Rodrigues et al., 2020).

Nessa perspectiva, a cartilha para a promoção de conforto aos familiares de pessoas em processo cirúrgico pode ser considerada como uma ferramenta válida e capaz de promover informações e orientações necessárias ao momento vivenciado pelo familiar.

Assim, o processo de validação de materiais educativos é considerado de fundamental importância para garantir a sua representatividade ao propor adaptações pertinentes. A validação desempenha papel importante na produção ou na melhoria da cartilha educativa, possibilitando melhor acolhimento (Wiesner et al., 2020). Além disso, o material educativo precisa promover a saúde e garantir que o cuidado seja oferecido ao público-alvo de forma eficaz, e para atingir esses objetivos, alterações realizadas mediante sugestão de juízes são necessárias (Leite et al., 2018).

Esse processo de adaptação do material educativo foi importante para tvrnar a tecnologia completa, com um alto rigor científico e que seja eficaz a prática dos profissionais de saúde (Vasconcelos et al., 2020), além de possibilitar orientações pertinentes ao processo de acompanhamento do familiar (Lima et al., 2017).

Nesse contexto, os achados do estudo, demostram que a cartilha pode auxiliar o processo de acompanhamento dos familiares que aguardam a cirurgia do seu ente, pois através dela ampliam seus conhecimentos e permanecem estáveis durante a realização do procedimento.

Importante ressaltar que as orientações prestadas verbalmente são de extrema importância para as pessoas que vão ser submetidas ao procedimento cirúrgico, bem como aos familiares que estão acompanhando, e precisam ser realizadas mesmo sendo fornecido materiais educativos. A disponibilidade de materiais educativos possibilita melhorar conhecimentos sobre a cirurgia e ser capaz de minimizar situações desconfortáveis, mas, atrelado a ela, o profissional de saúde, em especial o enfermeiro, precisa estar disponível para auxiliar nesse processo de acolhimento, a fim de que o mesmo seja ofertado de forma eficaz (Oliveira et al., 2016).

Vale ressaltar, que para a aplicabilidade da cartilha educativa, foi importante conhecer as possibilidades e limites para sua implementação aos familiares da pessoa no intraoperatório. Conforme os dados da pesquisa, as possibilidades da cartilha educativa, diz respeito a promoção e aceitação de informações relevantes aos familiares, além disso, a cartilha é uma ferramenta importante para promover conforto, distração e auxiliar no processo de ansiedade vivenciada. As cartilhas 
educativas são responsáveis pela disseminação de informações e orientações, que por vez, são capazes de facilitar o ensino e aprendizagem das pessoas, que muitas vezes se encontram em apreensão por informações, além disso, favorecem o cuidado, conforto, minimizando incertezas e desconfortos vivenciados (Silva et al., 2018; Varela et al., 2017).

Para os familiares da pessoa que se encontra no período intraoperatório, a cartilha educativa pode ser uma ferramenta importante por contribuir de forma favorável no processo de ansiedade vivenciado durante o período de espera, visto que, os familiares permanecem aguardando a cirurgia ansiosos por informações.

Como limitações da cartilha educativa, os achados apontam o nível de escolaridade dos familiares, o que levou a uma baixa taxa de concordância em diversos tópicos abordados na cartilha, o que pode comprometer a compreensão do material disponibilizado, uma vez que a linguagem abordada pode não estar apropriada para o público-alvo, além de encontrar pessoas analfabetas ou com um baixo nível de escolaridade. Nessa perspectiva, ao educar pessoas leigas e fornecer informações é essencial considerar as barreiras de alfabetização, além disso, é necessário que seja abordada uma linguagem de fácil entendimento, que atendam as capacidades de seus leitores e que sejam evitados vocabulários técnicos-científicos, pois impõe barreiras nos familiares (Wiesner et al., 2020; Wrench et al., 2019).

A linguagem abordada em um material educativo precisa ser clara e compreensível para seus leitores e adequada para qualquer público, como crianças e adolescentes. Ademais ao se tratar de um público leigo, é preciso evitar o uso de nomes técnicos, pois esses quesitos comprometem o entendimento das pessoas sobre o material, o consequentemente não atinge o objetivo proposto.

Além disso, os dados da pesquisa apontam em vários itens avaliados outras limitações presentes na cartilha educativa, que diz respeito a cartilha ser voltada para um único período cirúrgico, o intraoperatório, uma vez que, em todo o período perioperatório os familiares vivenciam sentimentos desagradáveis e precisam de conforto.

O período perioperatório expõe familiares a sentimentos e emoções desagradáveis, pois, ter um ente querido em hospitalização gera sofrimentos nos mesmos, em vista disso, fornecer informações sobre esse período alivia a ansiedade vivenciada, por isso deve ser o foco dos profissionais de saúde (Hamester et al., 2016).

Para os familiares que se encontram na sala de espera do CC, é relevante a abordagem do pós-operatório hospitalar, como em UTI, de forma que os familiares estejam preparados para enfrentar esse momento, caso seus entes não sejam liberados após a cirurgia.

A internação de uma pessoa na UTI após a realização da cirurgia é um momento que gera desequilíbrios emocionais, tanto para o paciente, quanto para seus familiares, que possuem um entendimento limitado sobre o ato cirúrgico e a necessidade de internação nessa unidade, tornando-os vulneráveis, e nesse contexto os familiares requerem uma atenção humanizada e que recebam informações pertinentes para esse momento (Milani et al., 2018).

Este estudo apresenta limitações referentes a impossibilidade de avaliar a cartilha com o público alvo, devido a pandemia da COVID-19, tendo em vista que na unidade de estudo, atividades e pesquisas foram suspensas, impossibilitando a ida da pesquisadora à referida unidade.

\section{Considerações Finais}

Os resultados deste estudo demonstram que a cartilha educativa, após cálculo da taxa de concordância, alterações e adaptações sugeridas pelos juízes, apresenta-se válida para os familiares da pessoa no período intraoperatório e será capaz de promover conforto e informações ao momento vivenciado no processo de acompanhamento da pessoa no período intraoperatório, quando for aplicada na prática.

Acreditamos que o estudo poderá contribuir para a educação em saúde dos familiares da pessoa no intraoperatório, permitindo que obtenham conhecimentos relevantes e permaneçam na sala de espera com conforto durante o processo de 
acompanhamento. Assim, esperamos que o material construído e validado seja disponibilizado para os familiares de pessoas em CC, para que possam enfrentar a espera da cirurgia com tranquilidade e bem-estar.

Faz-se necessário que outras pesquisas sejam realizadas visando o acolhimento e educação em saúde dos familiares que se encontram em processo de acompanhamento, pois os mesmos apresentam sentimentos desconfortáveis, tanto quanto os pacientes.

\section{Referências}

Bardin, L. (2016). Análise de conteúdo. Edições 70.

Bezerra, C. M., Cardoso, M. V. L. M. L., Silva, G. R. F., \& Rodrigues, E. C. (2018). Creation and validation of a checklist for blood transfusion in children. Rev Bras Enferm, 71(6), 3020-26. https://doi.org/10.1590/0034-7167-2018-0098.

Cardoso, R. B., Caldas, C. P., \& Souza, P. A. (2019). Nursing activities score e sua correlação com a teoria do conforto de Kolcaba: Reflexão teórica. Enferm Foco, 10(1), 87-92. https://doi.org/10.21675/2357-707X.2019.v10.n1.1347.

Domingues, E. A. R., Alexandre, N. M. C., \& Silva, J. V. (2016). Cultural adaptation and validation of the Freiburg Life Quality Assessment - Wound Module to Brazilian Portuguese. Rev Latino-Am Enfermagem, 24, e2684, 1-8. http://dx.doi.org/10.1590/1518-8345.0289.2684.

Escobar, M. A., Brewer, A., Caviglia, H., Forsyth, A., Jimenez-Yuste, V., Laudenbach, L., Lobet, S., McLaughlin, P., Oyesiku, J., Rodriguez-Merchan, E. C., Shapiro, A., \& Solimeno, L. P. (2018). Recommendations on multidisciplinary management of elective surgery in people with haemophilia. Haemophilia: the official journal of the World Federation of Hemophilia, 24(5), 693-702. https://doi.org/10.1111/hae.13549.

Hamester, L., Souza, E. N., Cielo, C., Moraes, M. A., \& Pellanda, L. C. (2016). Effectiveness of a nursing intervention in decreasing the anxiety levels of family members of patients undergoing cardiac surgery: a randomized clinical trial. Rev Latino-Am Enfermagem, 24, e2729, 1-8. https://doi.org/10.1590/15188345.0208.2729.

Jünger, S., Payne, S. A., Brine, J., Radbruch, L., \& Brearley, S. G. (2017). Guidance on Conducting and REporting DElphi Studies (CREDES) in palliative care: Recommendations based on a methodological systematic review. Palliative Medicine, 31(8), 684-706. https://doi.org/10.1177/0269216317690685.

Leite, S. S., Áfio, A. C. E., Carvalho, L. V., Silva, J. M., Almeida, P. C., \& Pagliuca, L. M. F. (2018). Construction and validation of an Educational Content Validation Instrument in Health. Rev Bras Enferm, 71(suppl 4), 1635-41. https://doi.org/10.1590/0034-7167-2017-0648.

Lima, A. C. M. A. C. C., Bezerra, K. C., Sousa, D. M. N., Rocha, J. F., \& Oriá, M. O. B. (2017). Development and validation of a booklet for prevention of vertical HIV transmission. Acta paul Enferm, 30(2), 181-9. https://doi.org/10.1590/1982-0194201700028.

Ministério da Saúde. (2012) Resolução no 466/12, de 12 de dezembro de 2012. Dispõe sobre pesquisa envolvendo seres humanos. Ministério da Saúde. http://bvsms.saude.gov.br/bvs/saudelegis/cns/2013/res0466_12_12_2012.html.

Milani, P., Lanferdini, I. Z., \& Alves, V. B. (2018). Caregivers' Perception When Facing the Care Humanization in The Immediate Postoperative Period From a Cardiac Surgery Procedure. J. res: fundam care Online, 10(3), 810-6. https://doi.org/10.9789/2175-5361.2018.v10i3.810-816.

Muniz, J. S., Freitas, K. S., Góis, J. A., Lima, A. B., Fontoura, E. G., \& Oliveira, M. A. N. (2019). Validation of a booklet designed to promote comfort of relatives of hospitalized patients. Rev Rene, 20, e41399, 1-9. https://doi.org/10.15253/2175-6783.20192041399.

Oliveira, A. P. A., Souza, E. M., \& Pellanda, L. C. (2016). Effectiveness of video resources in nursing orientation before cardiac heart surgery. Assoc Med Bras, 62(8), 762-7. http://dx.doi.org/10.1590/1806-9282.62.08.762.

Queiroz, T. A., Carvalho, F. P. B., Simpson, C. A., Barreto, E. L. F., \& Fernandes, A. C. L. (2015). Família: significado para os profissionais da estratégia de saúde da família. Rev Bras Promoç Saúde, 28(2), 274-80. https://doi.org/10.5020/18061230.2015.p274.

Ribeiro, L. C. C., Oliveira, T. C., Moreira, S., \& Paula, F. A. (2017). Construção e validação de manual sobre Burnout em professores. Revista de Enfermagem do Centro-Oeste Mineiro, 7, e1317, 1-9. https://doi.org/10.19175/recom.v7i0.1317.

Rodrigues, L. N., Santos, A. S., Gomes, P. P. S., Silva, W. C. P., \& Chaves, E. M. C. (2020). Construction and validation of an educational booklet on care for children with gastrostomy. Rev Bras Enferm, 73(3), 1-7. http://dx.doi.org/10.1590/0034-7167-2019-0108.

Silva, R. A., Ximenez, L. B., Cruz, A. G., Serra, M. A. A. O., Araújo, M. F. M., Andrade, L. M., Studart, R. M. B., \& Carvalho, Z. M. F. (2018). Sexual activity of people with spinal cord injury: development and validation of an educational booklet. Acta Paul Enferm, 31(3), 255-64. http://dx.doi.org/10.1590/1982-0194201800037.

Varela, A. I. S., Rosa, L. M., Radünz, V., Salum, N. C., \& Souza, A. I. J. (2017). Cartilha educativa para pacientes em cuidados paliativos e seus familiares: Estratégias de construção. Rev enferm UFPE online, 11(suppl 7), 2955-62. http://dx.doi.org/10.5205/1981-8963-v11i7a23476p2955-2962-2017.

Vasconcelos, V. M. S., Freitas, B. J. S. A., Martins, M. C. C., Oliveira, A. D. S, Viana, M. R. P., Mendes, C. M. M., \& Ramos, C. V. (2020). Validação de tecnologia educacional em saúde sobre "terapia nutricional enteral domiciliar" para cuidadores de idosos. Research, Society and Development, 9(10), e6149109062. https://doi.org/10.33448/rsd-v9i10.9062.

Wiesner, M., Zowalla, R., \& Pobiruchin, M. (2020). The Difficulty of German Information Booklets on Psoriasis and Psoriatic Arthritis: Automated Readability and Vocabulary Analysis. JMIR Dermatol, 3(1), e16095, 1-19. http://dx.doi.org/10.2196/16095. 
Research, Society and Development, v. 10, n. 2, e52410212836, 2021

(CC BY 4.0) | ISSN 2525-3409 | DOI: http://dx.doi.org/10.33448/rsd-v10i2.12836

Wrench, W., Van Dyk, L., Srinivas, S., \& Dowse, R. (2019). Outcome of illustrated information leaflet on correct usage of asthma-metered dose inhaler. Afr J Prim Health Care Fam Med, 11(1), e1-e9. https://doi.org/10.4102/phcfm.v11i1.2079.

Zhao, J., Gao, S., Wang, J., Liu, X., \& Hao Y. (2016). Differentiation between two healthcare concepts: Person-centered and patient-centered care. International Journal of Nursing Sciences. 3(4), 398-402. https://doi.org/10.1016/j.ijnss.2016.08.009. 